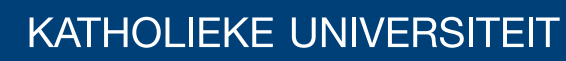 \\ LEUVEN
}

\section{Faculty of Business and Economics}

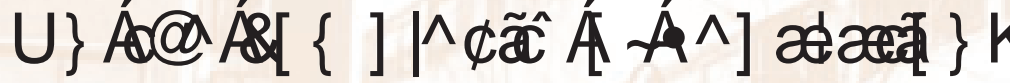
WAHMKL+HLQQH IDMJQP HQLSLREOP

7 UMNDP [' RMDW,RDQQQVIO RXLYPVIDQGG) UUN\&I5 M6 SLHMP D

DEPARTMENT OF DECISION SCIENCES AND INFORMATION MANAGEMENT (KBI) 


\title{
On the complexity of separation: the three-index assignment problem
}

\author{
Trivikram Dokka • Ioannis Mourtos • \\ Frits C.R. Spieksma
}

Received: date / Accepted: date

\begin{abstract}
A fundamental step in any cutting plane algorithm is separation: deciding whether a violated inequality exists within a certain class of inequalities. It is customary to express the complexity of a separation algorithm in $n$, the number of variables. Here, we argue that the input to a separation algorithm can be expressed in $|\sup (x)|$, where $\sup (x)$ denotes the vector containing the positive components of $x$. This input measure allows one to take sparsity into account. We apply this idea to two known classes of valid inequalities for the three-index assignment problem, and we find separation algorithms with a better complexity than the ones known in literature. We also show empirically the performance of our separation algorithms.
\end{abstract}

\section{Motivation}

Cutting plane algorithms constitute a fundamental way of solving combinatorial optimization problems. Typically, in such an approach, a specific combinatorial optimization problem is formulated as an Integer Linear Program (ILP)

This paper is an improved version of an extended abstract that appeared as "Fast separation algorithms for three index assignment problems" in the proceedings of ISCO 2012, LNCS 7422, pp. 189-200, 2012.

Trivikram Dokka

ORSTAT, K.U.Leuven, Naamsestraat 69, B-3000 Leuven, Belgium.

E-mail: Trivikram.Dokka@kuleuven.be

Ioannis Mourtos

Department of Management Science and Technology, Athens University of Economics and Business, 76 Patission Ave. 104334 Athens, Greece.

E-mail: mourtos@aueb.gr

Frits C.R. Spieksma

ORSTAT, K.U.Leuven, Naamsestraat 69, B-3000 Leuven, Belgium.

E-mail: frits.spieksma@kuleuven.be 
of the form $\min \left\{c^{T} x: A x=b, x \in Z_{+}^{n}\right\}$, where $x$ denotes an $n$-dimensional column vector of variables, and where $A, b$, and $c$ are given coefficients of appropriate dimensions. The convex hull of the feasible solutions is defined by the corresponding polyhedron $P_{I}=\operatorname{conv}\left\{x \in Z_{+}^{n}: A x=b\right\}$. Then, there is interest in identifying classes of inequalities that are valid for $P_{I}$ and 'strong enough' (for related background see, for example, Nemhauser and Wolsey [22]). Although identifying such families provides a (partial) characterization of $P_{I}$, the computational benefit of those families, in terms of finding $z=\min \left\{c^{T} x: x \in P_{I}\right\}$, can only be realized if those inequalities can be efficiently used to strengthen the linear programming relaxation $P_{L}=\min \left\{c^{T} x: A x=b, x \geq 0\right\}$.

Since there can be many inequalities within a family, the addition of inequalities, when employing a cutting plane methodology, should only be made 'on demand', i.e., an inequality is added to the current linear program only when it is violated by a specific vector $x \in \mathbb{R}^{n}$. The problem of determining whether such a vector violates an inequality of a specific family is called the separation problem for this family and an algorithm solving it is called a separation algorithm. Thus, an important task, once families of inequalities have been identified, is to design separation algorithms. These are often family-specific, and, in order to be useful, should be of low computational complexity.

It is quite customary (see Section 2 for examples) to express the complexity of a separation algorithm in terms of $n$, the dimension of the vector $x$. This seems reasonable since, at the very least, one would need to inspect each entry of the vector $x$ to decide whether a violated inequality exists. In fact, separation algorithms with a complexity of $O(n)$ have been called "best-possible" (Balas and Qi [4], Qi and Sun [24]). It is however important to realise that separation algorithms are normally incorporated within a Branch \& $C$ ut scheme, i.e., a search scheme to find $z=\min \left\{c^{T} x: x \in P_{I}\right\}$ by strengthening the LP-relaxation at various nodes of the search tree. Within such a scheme, a separation algorithm receives as input not any $x \in R^{n}$, but instead a vector describing an optimal solution of the current LP-relaxation, i.e., an $x \in P_{L} \backslash P_{I}$. Such an $x$ is typically sparse, meaning that relatively few entries are nonzero. Thus, we can describe $x$ by the support of $x, \sup (x)$, which is more compact than $x$ itself. This leads to the following question, which is the theme of our work: can we design better separation algorithms (in terms of worst-case complexity) by describing the computational complexity of a separation algorithm in terms of $|\sup (x)|$ (instead of the dimension of $x)$ ?

We will elaborate on this idea in Section 2. Of course, in practice, many separation algorithms already exploit sparsity. For instance, Applegate et al. [3] (and references therein), on their work on the TSP, describe how sparsity is used; another example is given in Kaparis and Letchford [19]; in Section 2 we come back to this issue, and give more examples. To the best of our knowledge, apart from work on TSP (see Subsection 2.1), no attempt has been made to express the complexity of a separation algorithm in terms of its input, the support of the vector $x$. We illustrate this idea on the (axial) three-index assignment problem (Section 4), and we show that "better-than-best-possible" 
separation algorithms are possible (Section 5). We also test empirically the performance of our separation algorithms on instances from literature; it turns out that the separation algorithms proposed here are much faster than the known ones (Section 6).

\section{An overview of the use of sparsity in separation algorithms}

There are many examples in literature where sparsity is used when separating a class of valid inequalities. In this section we describe briefly and informally a number of examples for various combinatorial optimization problems. In the rest of the section, $x$ denotes the incidence vector representing the solution of a given instance of the problem under consideration.

\subsection{The traveling salesman problem (TSP)}

The traveling salesman problem (TSP) is one of the most studied problems from a polyhedral point of view [3]. The following inequalities called sub-tour inequalities are well known for TSP polytope:

$$
\sum\left(x_{e}: e \text { has one end in } S \text { and one end not in } S\right) \geq 2
$$

for all nonempty proper subsets $S$ of cities.

To solve the separation problem for the subtour inequalities (1), the solution of the current LP relaxation, say $x^{*}$, is represented by building a support graph $G^{*}=\left(V^{*}, E^{*}\right)$. This graph $G^{*}$ has edges present only if the corresponding component in $x^{*}$ is positive. One of the heuristics (see page 159, [3]) used to solve the subtour separation problem is a parametric connectivity heuristic which only uses those edges in $G^{*}$ whose weight is more than a fixed $\epsilon>0$. In the Padberg-Rinaldi exact method a minimum cut in $G^{*}$ is computed to solve the subtour separation problem. Hence, in this approach, sparsity in the vector $x^{*}$ directly affects $G^{*}$. It follows that running times of methods that use $G^{*}$ depend on the sparsity of $x^{*}$.

Another important class of inequalities for the TSP polytope are comb and blossom inequalities. In [3], a separation heuristic called the odd component heuristic is used, which uses the graph $G^{*}$, whose vertex set is $V$ and whose edge set is $\left\{e \in E^{*}: 0<x_{e}^{*}<1\right\}$. Notice that the complexity of these algorithms is expressed in $\left|E^{*}\right|$, and hence the compact representation of the input is taken into account. More details on the separation algorithms for the traveling salesman problem can be found in [3] and Fleischer et al. [14].

\subsection{The stable set problem}

The stable set polytope of graph $G=(V, E)$ is defined as the convex hull of incidence vectors of all stable sets in $G$. It is denoted by 


$$
P_{S T A B}(G)=\operatorname{conv}\left\{\chi^{S} \mid S \subseteq V \text { stable set }\right\}
$$

The first class of well known valid inequalities of $P_{S T A B}(G)$ are the odd cycle inequalities.

$$
\sum_{i \in V^{\prime}} x_{i} \leq \frac{|C|-1}{2} \text { for each odd cycle } C=\left(V^{\prime}, E^{\prime}\right) \subseteq G
$$

To separate the odd-cycle inequalities (2) for a graph $G$ and a vector $x^{*}$, an auxiliary bipartite graph $H$ is constructed using fractional variables only, see Rebennack et al. [27]. A minimum weight odd cycle in $G$ is computed by finding a shortest path in $H$. For more details and description of the algorithms see [27], Cheng and Cunningham $[10,11]$ who give a separation algorithm with complexity $O(|V| \cdot|E| \cdot \log |V|)$.

\subsection{The clique partitioning problem}

The clique partitioning problem is a problem defined for a graph $G=(V, E)$, see Grötschel and Wakabayashi [15]. Here sparsity is also used in separation algorithms for a class of valid inequalities for a formulation of the clique partitioning problem. They prove that the so-called partition inequalities are facet defining for the clique partitioning polytope in [16]. To (heuristically) separate the partition inequalities, in [15] they employ an algorithm that only use the positive components of the vector $x^{*}$ which has complexity of $O\left(|V|^{3}\right)$. More details for separation problems for clique partitioning problem can be found in Letchford and Sørenson [21], and Oosten et al. [23], [15].

\subsection{The set partitioning problem}

The set partitioning problem is a fundamental problem in combinatorial optimization, see Balas and Padperg [7] for a survey. Formally the set partitioning problem is defined as follows:

$$
\min \left\{c x \mid A x=e, x_{i}=0 \text { or } 1, \forall j \in N\right\}
$$

where $A$ is an $m \times n$ matrix of zeros and ones, $c$ is an arbitrary $n$-vector, $e=$ $(1, \ldots, 1)$ is an $m$-vector, and $N=\{1, \ldots, n\}$. We denote the set partitioning polytope by $P_{S P P}$. It is proven in [7] that the following inequalities are facet defining for $P_{S P P}$ :

$$
\sum_{j \in K} x_{j} \leq 1
$$

where $K \subseteq N, K$ is the node set of a clique in $G_{A}$ (intersection graph of $A$ ), and: 


$$
\sum_{j \in G^{\prime}} x_{j} \leq \frac{\left|G^{\prime}\right|-1}{2}
$$

where $G^{\prime}$ (a subgraph of $G_{A}$ ) is an odd hole.

The inequalities (3) and (4) are called clique and odd hole inequalities respectively.

Caprara and Salazar [9] formulate the index selection problem as a set packing problem, and they solve the separation problem for odd hole inequalities by determining a minimum weight odd cycle in a graph having one node for each fractional variable, where each edge $(i, j)$ is given the weight $\left(1-x_{i}^{*}-x_{j}^{*}\right)$. If the minimum weight odd cycle has weight lower than 1 , then a violated odd hole inequality is found.

Ropke et al. [28] formulate the pickup and delivery problem with time windows as a set partitioning problem. They heuristically solve the separation problem for the so-called rounded capacity inequalities by only considering the positive values in the input vector.

In Escudero et al. [13], the winner determination problem for combinatorial auctions is formulated as a set packing problem and a branch-and-cut algorithm is presented. They develop separation procedures which use only fractional components of $x^{*}$.

\subsection{Other occurrences of sparsity}

In Kececioglu et al. [20] a branch-and-cut algorithm is presented for sequence alignment problems. Separation procedure is outlined based on a graph which is constructed from the fractional variables in the input vector.

Van den Akker et al. [26] use a branch-and-cut approach to solve the timeindexed formulation for single machine scheduling problems. In their separation algorithms they only use the fractional components of the input vector.

Alvarez- Valdes et al. [1] formulate the pallet loading problem as a 0-1 integer program and propose a branch-and-cut algorithm. In their separation procedure they use a graph; they reduce the size of this graph by having a vertex correponding to each fractional variable whose value exceeds a certain threshold.

These are all examples of cases where sparsity is used to speed up the separation procedure. However, in most of these cases the focus is on solving instances of the underlying optimization problem, and hence the complexity of a separation algorithm is measured in $n$. We now propose an alternative.

\section{The idea}

In Section 2 we have seen examples where sparsity is implicitly used in a separation algorithm. In this section, we propose a viewpoint taking sparsity explicitly into account. 
Traditionally, to describe the input needed for a specific class of inequalities, the $\mathrm{n}$ entries of the vector $x=\left(x_{1}, \ldots, x_{n}\right)$ were given. We propose an alternative measure of the input: the cardinality of the support of the vector $x$, denoted by $T:=|\sup (x)|$. Thus, we propose to express the running time of a separation algorithm in the number of nonzero entries of the vector $x$, denoted by $T$. This idea allows us to make use of the fact that, typically, the input a separation algorithm receives is not just some vector $x$; instead it is almost always the extreme point of a polyhedron, and hence, it typically has few nonzero entries. Hence the number of computations of a separation algorithm does not depend on the size of the original instance, but rather on the number of non-zero entries in the $x$-vector. Indeed, we emphasize here that our viewpoint is restricted to exclusively solving instances of the separation problem; here, we are not interested in solving the underlying optimization problem.

In order to further illustrate this point of view, consider the following (imaginary) setting. Suppose that many different instances of some combinatorial optimization problem are being solved through a cutting-plane approach using multiple computers in parallel. However, there is only a single computer available that is able to run a separation routine for a specific class of valid inequalities. Then, this routine will receive frequently fractional vectors corresponding to the instances being solved by the cutting-plane approach, and it will return each time a violated inequality provided one exists. In such a situation, the value of $n$ is not explicitly inputted; indeed, the sole input are the positive components of the vector $x$, i.e., the support of $x$ : $\sup (x)$. Therefore, we propose in this work to express the number of operations needed by the separation routine in terms of the length of the support of $x$, i.e., in $T:=$ $|\sup (x)|$.

Thus, we view the decision problem with respect to a certain class of valid inequalities $C$, as follows

\section{- INSTANCE: support $(x)$}

- QUESTION: Does there exist a violated inequality within $C$ ?

Obviously, the setting described above is hypothetical. However, the point we want to emphasize is that it is not so much solving an instance of some combinatorial optimization problem that matters to us. What matters is to answer the decision question formulated above as efficiently as possible. We use the traditional tools of complexity of algorithms, namely to express the number of operations in the length of the input, and indeed the input to a separation problem is nothing more than the support of a vector $x$.

In the next section, we describe the 3 -index assignment polytope. 


\section{The 3-index assignment polytope}

The 3 -index assignment problem, defined on three disjoint $n$-sets $I, J, K$ and a weight function $w: I \times J \times K \longrightarrow \mathbb{R}$, asks for a collection of triples $M \subseteq$ $I \times J \times K$ such that each element of any set appears in exactly one triple, and the function $w$ is minimized (over all possible such collections). Its formulation as an ILP is

$$
\begin{array}{ll}
\min & \sum_{i \in I} \sum_{j \in J} \sum_{k \in K} w_{i j k} x_{i j k} \\
\text { s.t. } & \sum_{j \in J} \sum_{k \in K} x_{i j k}=1 \quad \forall i \in I, \\
& \sum_{i \in I} \sum_{k \in K} x_{i j k}=1 \quad \forall j \in J, \\
& \sum_{i \in I} \sum_{j \in J} x_{i j k}=1 \quad \forall k \in K, \\
& x_{i j k} \in\{0,1\} \quad \forall i \in I, j \in J, k \in K .
\end{array}
$$

Let $A^{n}$ denote the $(0,1)$ matrix corresponding to the constraints $(5)-(7)$, which has $n^{3}$ columns and $3 n$ rows. Notice that from this point in the text onwards, $n$ denotes the cardinality of each set being 'assigned'; hence, the number of variables is $n^{3}$. Then, the 3 -index assignment polytope is $P_{I}^{n}=\operatorname{conv}\{x \in$ $\left.\{0,1\}^{n^{3}}: A^{n} x=e\right\}$, while its LP-relaxation is $P^{n}=\left\{x \in R^{n^{3}}: A^{n} x=e, x \geq\right.$ $0\}$. For a survey on the 3 -index assignment problem, see Spieksma [25].

The investigation of the facial structure of the polytope $P_{I}$ was initiated by Balas and Saltzman [5]. We will now describe two families of facet-defining inequalities that were originally described in [5]. See Appa et al. [2] for the $k$ index case. The column intersection graph of $A^{n}$, namely $G(V, E)$, has a node for each column of $A^{n}$ and an edge for every pair of columns that have a +1 entry in the same row. Notice that a column contains three +1 's. We define the intersection of two columns $c$ and $d$ as the set of rows of $A^{n}$ such that each row in the set has a +1 entry in column $c$ and in column $d$; this is denoted by $|c \cap d|$. It is easy to see that $V=I \times J \times K$ and $E=\{(c, d):\{c, d\} \subseteq V,|c \cap d| \geq 1\}$, i.e., a node in $V$ corresponds to a triple, and two nodes are connected if the corresponding triples share some index. A clique is a maximal complete subgraph.

In $G(V, E)$, there are two types of cliques that give rise to families of inequalities that are known to be facet-defining for $P_{I}$. To formally define the two types of clique inequalities, let:

- for each $c \in V: Q(c)=\{d \in V:|c \cap d| \geq 2\}$,

- for each $c \in V: \operatorname{co} Q(c)=\{d \in V:|c \cap d|=1\}$, and

- for each $c, d \in V$ with $|c \cap d|=0: Q(c, d)=\{c\} \cup\{Q(d) \cap \operatorname{co} Q(c)\}$.

Thus, $Q(c)$ is the set of triples sharing at least two indices with triple $c$, while $\operatorname{co} Q(c)$ is the set of triples that has exactly one index in common with triple $c$. Finally, when given two disjoint triples $c$ and $d, Q(c, d)$ is the set of triples that has two indices in common with $d$, and one with $c$, together with 
triple $c$. Notice that $Q(c, d)$ has exactly four elements. As usual, we write $x(A)$ for $\sum_{q \in A} x_{q}$.

Definition 1 For each $c \in V$, the facet-defining inequality $x(Q(c)) \leq 1$ is called a clique inequality of type $I$.

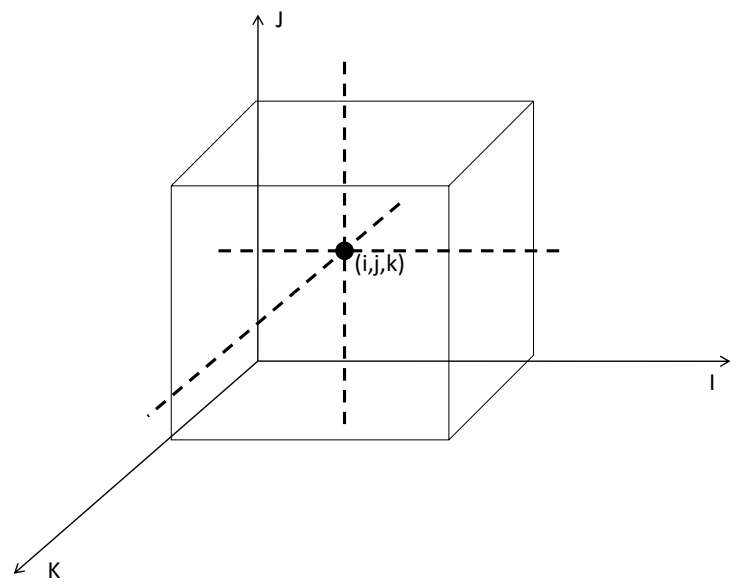

Fig. 1 Geometric illustration of a clique inequality of type I: the sum of $x$ values of the triples on the dotted lines corresponds to the lefthand side of a clique inequality of type $I$.

When we organize the variables $x_{i j k}$ in a three-dimensional array (a cube), a clique inequality of type I can be seen as the sum of those $x$-variables that lie on the three "axes" through a particular cell (see Figure 1 for a geometric illustration).

Definition 2 For each $c, d \in V$ with $|c \cap d|=0$, the facet-defining inequality $x(Q(c, d)) \leq 1$ is called a clique inequality of type $I I$.

An illustration of a clique inequality of type II is given in Figure 2.

Separation of cliques of type I and II has first been treated in Balas and Saltzman [5] through algorithms of $O\left(n^{4}\right)$ time complexity. Improved $O\left(n^{3}\right)$ algorithms (i.e., of complexity linear in the number of variables) have been presented in Balas and Qi [4]. These separation algorithms are called 'bestpossible'. In the next section we show how these complexities can be improved when we use as input-measure the support of the given vector $x$. As motivated in Section 3, this measure of input makes sense in a cutting-plane context where each iteration amounts to solving a linear program. 


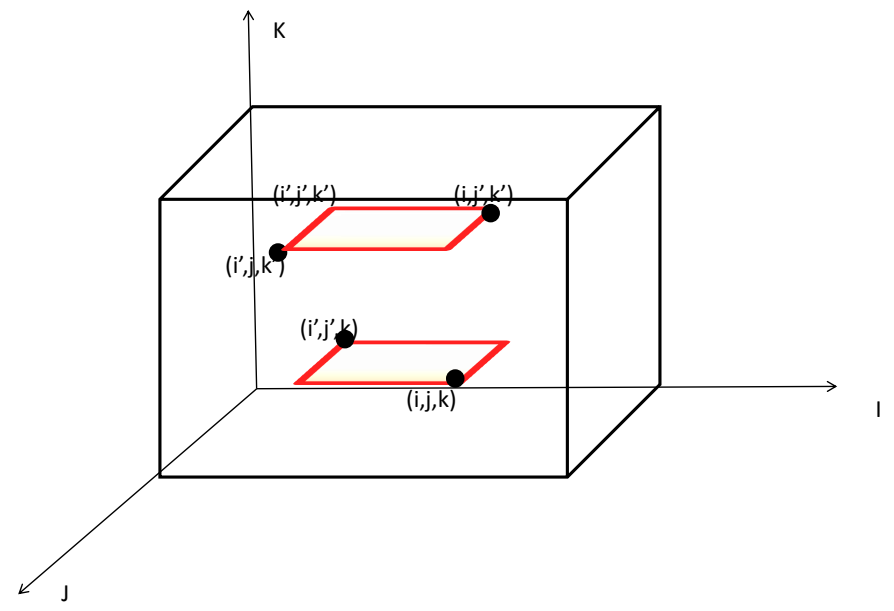

Fig. 2 Geometric illustration of a clique inequality of type II: the sum of $x$ values of points corresponds to the left hand side of a clique inequality of type I.

The following fact from linear programming theory is well-known: the number of nonzero variables in an extreme point does not exceed the number of constraints. As a consequence, we have:

Remark 1 An extreme point of $P^{n}$ has at most $3 n$ non-zero variables.

Given an arbitrary vector $x \in P^{n}$, let $\sup (x)$ be a list of elements (or triples) with positive values in $x$, i.e., $\sup (x)=\left\{c \in V: x_{c}>0\right\}$ and let $T=|\sup (x)|$. Thus, $T$ is the number of positive elements in $x$. We assume - as described in Section 3 - that a vector $x \in P^{n}$ is described by $\sup (x)$ containing the triples, and the corresponding (positive) fraction. This corresponds, for instance, to a basic solution from an LP-solver. We further assume that the input vectors received by the separation algorithm are elements of $P^{n}$ i.e., we assume that they satisfy (5) - (7). It follows that $T \geq n$, since any solution in $P^{n}$ has at least $n$ positive values in the solution vector $x$. In the next section we provide $O(T)$ separation algorithms for clique inequalities of type I and type II.

\section{Separating clique inequalities of type I and type II}

We provide an $O(T)$ separation algorithm for the clique inequalities of type I (Subsection 5.1), and an $O(T)$ separation algorithm for the clique inequalities of type II (Subsection 5.2). 
5.1 Separation of clique inequalities of type I

We now proceed with some preliminaries. Consider the following quantities. Define, for $x \in P^{n}$ :

- for each $j \in J, k \in K: \operatorname{SUM} I(j, k)=\sum_{i \in I} x_{i j k}$,

- for each $i \in I, k \in K: \operatorname{SUM} J(i, k)=\sum_{j \in J} x_{i j k}$,

- for each $i \in I, j \in J: \operatorname{SUM} K(i, j)=\sum_{k \in K} x_{i j k}$.

Informally, each of these quantities corresponds to an axis in the geometric description given in Figure 1.

Further, we define

- for each $i \in I: J(i)=\left\{j \in J: \operatorname{SUM} K(i, j)>\frac{1}{3}\right\}$.

Notice that $|J(i)| \leq 2$ for each $i \in I$, since inequalities (5) hold.

By scanning once through $\sup (x)$, we can compute all these quantities; see Algorithm 1 for a precise description. Thus, we can state the following Lemma:

Lemma 1 For an arbitrary $x \in P^{n}$, Algorithm 1 calculates in $O(T)$ steps all positive $\operatorname{SUMI}(j, k), \operatorname{SUMJ}(i, k)$ and $\operatorname{SUMK}(i, j)$ values (for $i \in I, j \in J, k \in$ $K)$ as well as the sets $J(i)$ for each $i \in I$.

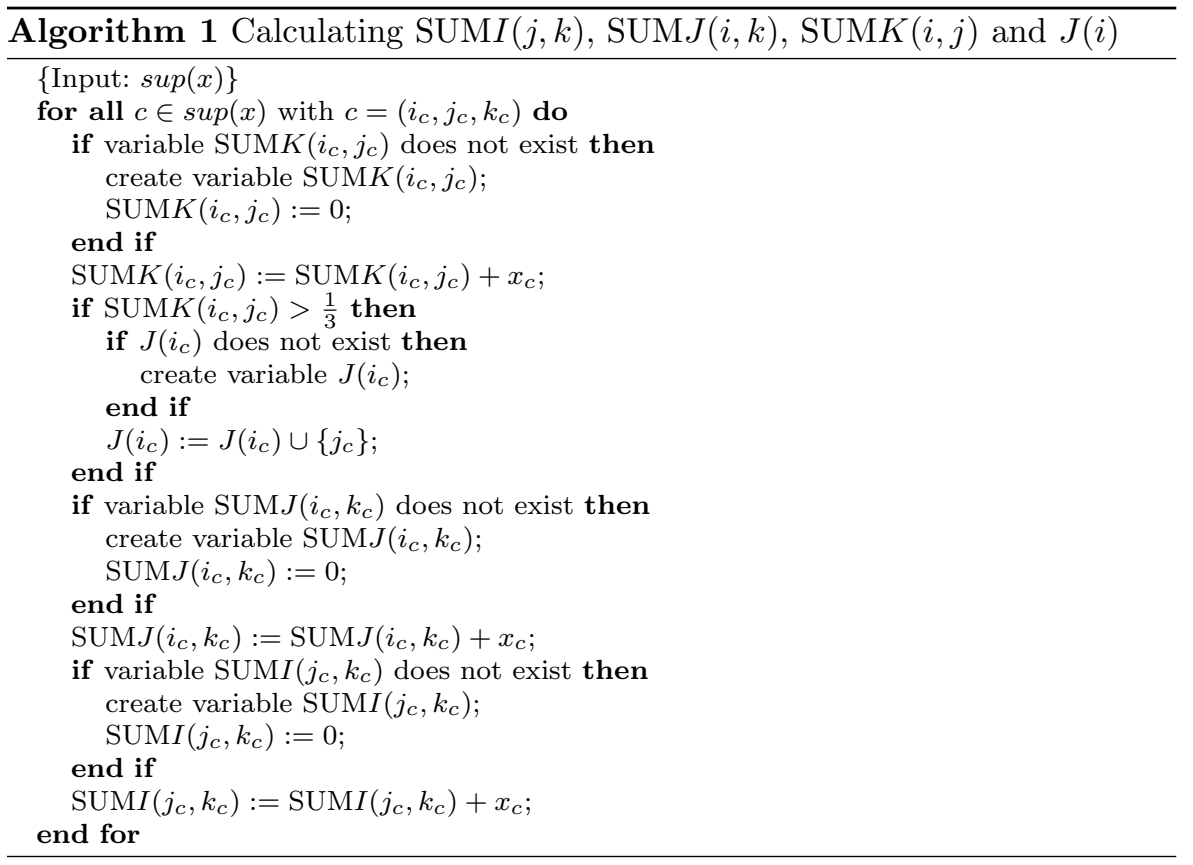

We now provide an $O(T)$ separation algorithm for clique inequalities of type I. Recall that, with $c=\left(i_{c}, j_{c}, k_{c}\right), Q(c)=\left\{\left(i_{c}, j_{c}, k_{c}\right)\right\} \cup\left\{\left(i_{c}, j_{c}, k\right), k \in\right.$ 
$\left.K \backslash\left\{k_{c}\right\}\right\} \cup\left\{\left(i_{c}, j, k_{c}\right), j \in J \backslash\left\{j_{c}\right\}\right\} \cup\left\{\left(i, j_{c}, k_{c}\right), i \in I \backslash\left\{i_{c}\right\}\right\}$ (see Figure 1). Thus:

$$
x(Q(c))=\operatorname{SUM} K\left(i_{c}, j_{c}\right)+\operatorname{SUM} J\left(i_{c}, k_{c}\right)+\operatorname{SUM} I\left(j_{c}, k_{c}\right)-2 \cdot x_{i_{c} j_{c} k_{c}} .
$$

In the rest of the section we will assume that a violated inequality (if it exists) corresponding to a triple $(i, j, k)$ satisfies: $\operatorname{SUM} K(i, j) \geq \operatorname{SUM} J(i, k) \geq$ $\operatorname{SUM} I(i, k)$. Notice that this assumption is without loss of generality since we can interchange the role of $i, j, k$. Algorithms similar to this case can be constructed for the other cases by interchanging the role of $i, j, k$ and precomputing the sets similar to $J(i)$ using Algorithm 1.

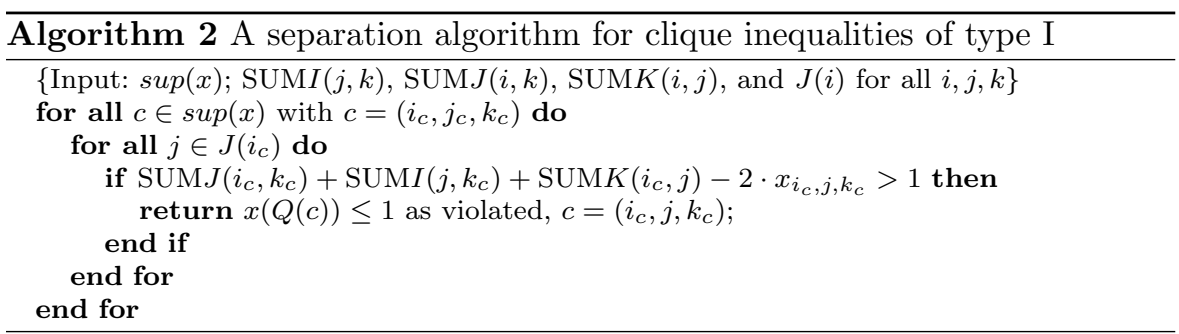

Lemma 2 Given its input, Algorithm 2 determines in $O(T)$ steps whether an arbitrary $x \in P^{n}$ violates a clique inequality of type $I$.

Proof Notice that a clique inequality of type I is violated only if there exists $\left(i_{c}, j_{c}, k_{c}\right)$ such that

$$
\operatorname{SUM} K\left(i_{c}, j_{c}\right)>\frac{1}{3}
$$

and

$$
\operatorname{SUM} J\left(i_{c}, k_{c}\right)>0 \text {. }
$$

That is, (10) follows directly from (9) and our assumption that $\operatorname{SUM} K\left(i_{c}, j_{c}\right) \geq$ $\operatorname{SUM} J\left(i_{c}, k_{c}\right) \geq \operatorname{SUM} I\left(j_{c}, k_{c}\right)$. Concerning (11), observe that $\operatorname{SUM} J\left(i_{c}, k_{c}\right)=$ 0 yields $\operatorname{SUM} I\left(j_{c}, k_{c}\right)=0$, while the remaining terms of (9) cannot sum to a total of more than 1 and hence (9) cannot be violated.

Observe that, each $\left(i_{c}, k_{c}\right) \in I \times K$ satisfying (11) is contained in some triple in $\sup (x)$. Algorithm 2 proceeds by considering each triple in sup $(x)$. Once $i_{c}, k_{c}$ are fixed, then by definition all $j_{c}$ for which (10) is satisfied are stored in $J\left(i_{c}\right)$ which has been pre-calculated. Algorithm 2 proceeds by checking the inequality for each $\left(i_{c}, j, k_{c}\right)$ with $j \in J\left(i_{c}\right)$ after $i_{c}, k_{c}$ are fixed. Hence a violated inequality is found if there exists one.

Regarding complexity, the first 'for' loop runs $O(T)$ times. The second 'for' loop runs for $O(1)$ times as the cardinality of $J(i)$ is at most 2 . Therefore, the overall complexity of Algorithm 2 is $O(T)$. 
Theorem 1 For any $x \in P^{n}$, clique inequalities of type I can be separated in $O(T)$ steps.

Proof Lemma's 1 and 2 imply that first applying Algorithm 1, and then applying Algorithm 2, determines whether there is a violated inequality of type I. Total complexity follows from the complexities of these algorithms.

5.2 Separation of clique inequalities of type II

Let $\operatorname{val}(i)(\operatorname{val}(j), \operatorname{val}(k))$ be the value of the third largest variable indexed by a triple containing $i(j, k)$, for each $i \in I(j \in J, k \in K)$. Then we can define for any $x \in P^{n}$ :

- for each $i \in I: A(i)=\left\{(i, j, k) \in V: x_{i j k} \geq \operatorname{val}(i)\right\}$,

- for each $j \in J: B(j)=\left\{(i, j, k) \in V: x_{i j k} \geq \operatorname{val}(j)\right\}$,

- for each $k \in K: C(k)=\left\{(i, j, k) \in V: x_{i j k} \geq \operatorname{val}(k)\right\}$.

Notice that the sets $A(i)(B(j), C(k))$ contain the largest three such variables (i.e., variables indexed by a triple containing $i(j, k))$. In addition, we define

- for each $i \in I: A^{>\frac{1}{4}}(i)=\left\{(i, j, k): x_{i j k}>\frac{1}{4}, j \in J, k \in K\right\}$,

- for each $j \in J: B^{>\frac{1}{4}}(j)=\left\{(i, j, k): x_{i j k}>\frac{1}{4}, i \in I, k \in K\right\}$,

- for each $k \in K: C^{>\frac{1}{4}}(k)=\left\{(i, j, k): x_{i j k}>\frac{1}{4}, i \in I, j \in J\right\}$.

Observe further that all elements from a specific $A^{>\frac{1}{4}}(i)$ (or $B^{>\frac{1}{4}}(j)$ or $C^{>\frac{1}{4}}(k)$ ) occur in a single equality from constraints (5) (or (6), or (7)). Since the right-hand side of this equality equals 1 , and since each element in the set has weight at least $\frac{1}{4}$, it follows that $\left|A^{>\frac{1}{4}}(i)\right| \leq 3,\left|B^{>\frac{1}{4}}(j)\right| \leq 3$, and $\left|C^{>\frac{1}{4}}(k)\right| \leq 3$, for $i \in I, j \in J, k \in K$..

Again, scanning $\sup (x)$ allows us to compute the sets defined above (see Algorithm 5 for a precise description).

Lemma 3 For an arbitrary $x \in P^{n}$, Algorithm 5 calculates in $O(T)$ steps the sets $A(i), B(j), C(k), A^{>\frac{1}{4}}(i), B^{>\frac{1}{4}}(j)$ and $C^{>\frac{1}{4}}(k)$ for $i \in I, j \in J, k \in K$.

Proof Since each element of $\sup (x)$ is inspected once, the $O(T)$ complexity follows easily.

Let us now focus on clique inequalities of type II. For $c=\left(i_{c}, j_{c}, k_{c}\right)$ and $h=\left(i_{h}, j_{h}, k_{h}\right), Q(h) \cap \operatorname{co} Q(c)=\left\{\left(i_{c}, j_{h}, k_{h}\right),\left(i_{h}, j_{c}, k_{h}\right),\left(i_{h}, j_{h}, k_{c}\right)\right\}$. Hence $Q(c, h)=\{c\} \cup(Q(h) \cap \operatorname{co} Q(c))$ is a clique with exactly four nodes, any pair of which share exactly one index; notice also that any node in $Q(c, h)$ can play the role of $c$. Last, observe that any three nodes in $Q(c, h)$ are a subset of the node set of a clique of type I; for example, $\left\{\left(i_{c}, j_{h}, k_{h}\right),\left(i_{h}, j_{c}, k_{h}\right),\left(i_{h}, j_{h}, k_{c}\right)\right\} \subseteq$ $Q(h),\left\{\left(i_{c}, j_{c}, k_{c}\right),\left(i_{c}, j_{h}, k_{h}\right),\left(i_{h}, j_{c}, k_{h}\right)\right\} \subseteq Q\left(\left(i_{c}, j_{c}, k_{h}\right)\right)$, and so on.

Assuming that no clique inequality of type $\mathrm{I}$ is violated, i.e., Algorithm 2 has returned no violated inequality, we provide an $O(T)$ separation algorithm for clique inequalities of type II. 


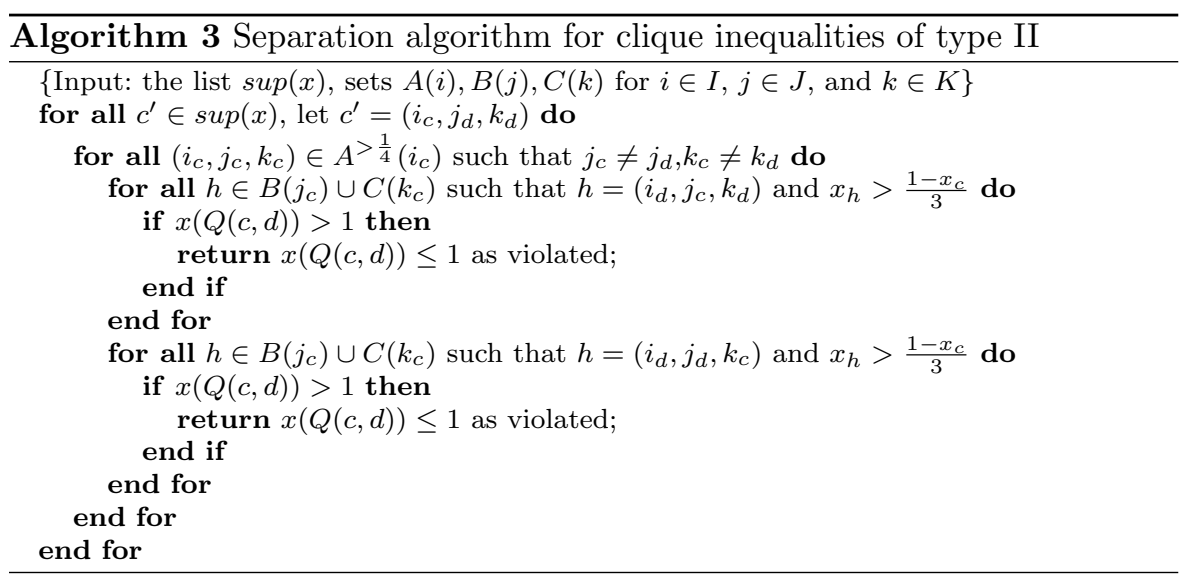

Lemma 4 Given its input, Algorithm 3 determines in $O(T)$ steps whether an arbitrary $x \in P^{n}$ violates a clique inequality of type II.

Proof Let us first examine the correctness of the algorithm, by considering some $x \in P^{n}$ such that

$$
x(Q(c, d))=x_{i_{c} j_{d} k_{d}}+x_{i_{d} j_{c} k_{d}}+x_{i_{d} j_{d} k_{c}}+x_{i_{c} j_{c} k_{c}}>1 .
$$

Recall that (12) is symmetric in the sense that any three variables in it appear together in a clique inequality of type I and any two variables in it have exactly one index in common. Therefore, let us assume without loss of generality that

$$
x_{i_{c} j_{c} k_{c}} \geq \max \left\{x_{i_{c} j_{d} k_{d}}, x_{i_{d} j_{c} k_{d}}, x_{i_{d} j_{d} k_{c}}\right\} .
$$

Then, the triple indexing the variable with the smallest value in (12) can be $\left(i_{c}, j_{d}, k_{d}\right)$ or $\left.\left(i_{d}, j_{c}, k_{d}\right)\right)$ or $\left(i_{d}, j_{d}, k_{c}\right)$; it suffices to prove the correctness of the algorithm for the case where $x_{i_{c} j_{d} k_{d}}$ is the smallest of the four variables in (12). Due to the assumption that no clique inequality of type I is violated, it follows that all four variables from (12) must be positive, and hence appear in $\sup (x)$. Therefore, $\left(i_{c}, j_{d}, k_{d}\right) \in \sup (x)$. Algorithm 3 proceeds by considering each possibility of $\left(i_{c}, j_{d}, k_{d}\right)$ in $\sup (x)$. Further, observe that at least one of the variables of (12) must have a value greater than $\frac{1}{4}$. By (13) we have $x_{i_{c} j_{c} k_{c}}>\frac{1}{4}$, for some $\left(i_{c}, j_{c}, k_{c}\right) \in \sup (x)$. Note that for a fixed $i_{c}$ such an $\left(i_{c}, j_{c}, k_{c}\right)$ should be in $A^{>\frac{1}{4}}\left(i_{c}\right)$. Moreover, for any fixed $c=\left(i_{c}, j_{c}, k_{c}\right)$ such that $x_{c}>\frac{1}{4}$, there are at most 4 possible triples $h$ that do not contain $i_{c}$, and $x_{h}>\frac{1-x_{c}}{3}$. By definition they all should be in $B\left(j_{c}\right)$ and $C\left(k_{c}\right)$. Consequently, Algorithm 3 is correct.

Concerning the complexity of the algorithm, note that there are four loops, namely one 'outer', one 'inner' and two 'innest' (with a slight language abuse). The 'outer' loop is performed $O(T)$ times. Since the cardinality of $A^{>\frac{1}{4}}(i), B^{>\frac{1}{4}}(j), C^{>\frac{1}{4}}(k)$ is at most 3 , the inner loop is performed only 3 times at most. Therefore 'inner' loop runs for $O(T)$ times. For each $c \in \sup (x)$ such that $x_{c}>\frac{1}{4}$, the two 
'innest' loops are performed a constant number of times as the cardinality of $A\left(i_{c}\right), B\left(j_{c}\right), C\left(k_{c}\right)$ is constant. Therefore, the two 'innest' loops are run $O(T)$ times. In total, the complexity of the algorithm is $O(T)$.

Theorem 2 For any $x \in P^{n}$, clique inequalities of type II can be separated in $O(T)$ steps.

Proof Lemma's 3 and 4 imply that first applying Algorithm 5, and then applying Algorithm 3 determines whether there is a violated inequality of type II.

We close this section by mentioning the following. Other facet-defining inequalities than the clique inequalities discussed here, exist for the 3-index assignment polytope. In particular, so-called lifted 5-hole inequalities are known, see [5]. Using ideas described in this section we can design an $O(T)$ separation algorithm for this class of inequalities. Finally, we point out that the above $O(T)$ algorithms can be generalized to separate the inequalities that generalize clique inequalities of type I and II to the $k$-index case, $k \geq 3$.

\section{Computational experiments}

In this section we report on the computational performance of the algorithms presented in Subsection 5.1 for cliques of type I, and in Subsection 5.2 for cliques of type II. We compare the running times of our algorithms with the performance of the traditional separation algorithms described in [4]. All algorithms have been coded in $\mathrm{C}++$ using Visual Studio $\mathrm{C}++2005$ and ILOG concert technology; all the experiments are run on a Dell Latitude E6400 personal computer with Intel core 2 Duo processor with $2.8 \mathrm{Ghz}$ clock speed and 1.59 GB RAM, equipped with Windows XP. CPLEX 10.2 was used for solving the linear programs.

\subsection{Instances}

We took four classes of instances from the literature in order to compare the running times of the algorithms. The first class of instances was described in Balas and Saltzmann [6]; integer cost coefficients $c_{i, j, k}$ are generated uniformly in the interval $[0,100]$. We limited our experiments to the largest instances, namely those of size $n=24, n=26$; There are 10 instances, results are given in Table 1

The second class of instances are those described by Crama and Spieksma [12]. There are in total 18 instances, 9 with size $n=66$, and 9 with size $n=33$, see [12] for a precise description of the generation of these instances. From these 18 instances, 7 did not possess any violated inequalities of type I or type II. Hence, we only report computation times for the remaining 11 instances. Results are given in Table 2 . 
The third class of problems is described in Höfler and Fügenschuh [18] which consists of 4 subclasses: the first subclass of problems is created using the technique described in the Burkard et al. [8] consisting of problems of sizes $n=25,54,66,80$. These 4 instances are referred with a prefix brw. The second subclass of problems is created using the technique employed in [6] but with sizes $n=25,54,66,80$, and with the cost coefficients in the interval $[0,9999]$. These instances are prefixed with uniform $10 k$. Results for these two subclasses are found in Table 3 . The third subclass of problems is generated randomly with cost coefficients having a value $10000 \cdot z^{2}$ with $z$ in the interval $[0,1]$. These instances are referred with a prefix quad. The fourth subclass is called cluster class with cost coefficients chosen out of three clusters $[0,49],[450,499],[950,999]$ with the probability of a generated number to lie in a specific cluster was $1 / 3$ for each cluster, and numbers in each cluster are chosen uniformly. These instances are referred with a prefix cluster. Results for subclasses three and four are given in Table 4 .

The last class has two instances of size $n=100$ taken from Grundel and Pardalos [17] (see Table 5).

Notice that other instances are available, in particular instances described by Burkard et al. [8]. However, running times of the separation algorithms are negligible, which is caused by the size of these instances $(n \leq 16)$. We choose not to report these instances.

\subsection{Implementation details}

There are different ways of implementing a cutting plane algorithm. For instance, one can add a single violated inequality in each iteration, or one can add all violated inequalities in an iteration. We experimented with both options. Here, however, we only report results of the implementation where all violated inequalities are added. The reason for this is that when adding a single inequality the inequality found by our separation algorithms may differ from the violated inequality found by the traditional algorithms. This may lead to a different number of iterations, and to a less transparent comparison. We point out that the computational results for any setting we experimented with, show the same pattern.

Further, we have opted for the following strategy: first, find out whether violated inequalities of type I exist, if yes: add them to the linear program and re-optimize, else find out whether violated inequalities of type II exist, see Algorithm 4 for a precise description. Of course, this procedure favors the detection of violated type I inequalities.

\subsection{Results and Discussion}

The outcomes of our experiments are described in Tables 1-2. We denote our separation algorithms with Fast-sep. The first and second columns specify the 


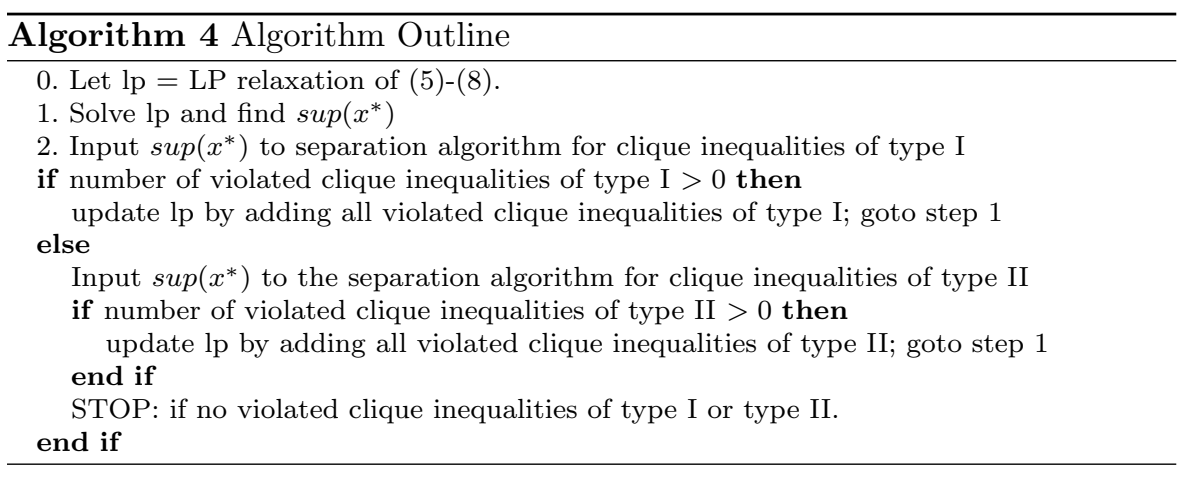

name and size of the instance. The third to seventh column give iteration number, LP objective value, number of violated inequalities, separation time using Fast-sep, and separation time using traditional algorithms at each iteration respectively. The type of the violated inequality found is indicated in brackets in column 4. All computation times are expressed in seconds.

As can be seen in the Tables 1-2, our algorithms outperform the existing separation algorithms in all the cases. Thus, not only theoretically, also in practice the separation algorithms presented here are faster than the traditional ones, often by an order of magnitude. On average, time needed by Fast-sep is less than $10 \%$ of the time needed by the algorithm from [4]. Moreover, it should be noted that as the size of the instances grows the increase in running times of the traditional algorithms is much larger compared to our algorithms. An interesting observation is that violated type II inequalities are rare; this may be caused (partly) by the setup of the separation routine. Notice further that the final solution for these instances was still fractional, except for two instances. Thus, only adding violated inequalities of type I and II is usually not enough to solve these instances, as also can be seen from the modest effect on the objective value.

Of course, one might be interested in comparing the separation times with the time needed to (re)solve the LP. This can be seen in Table 6 , where we focus on all instances with size $n \geq 54$. The last column depicts the time spent on solving the LP, except for the first iteration where model (5)-(7) is solved from scratch. It can be seen that the time spent in separation is significant with respect to time spent on re-optimizing the LP.

\section{Conclusion}

In this work, we have described a novel idea concerning the design of separation algorithms. By allowing the vector $x$ to be described by its support, we obtained more efficient separation algorithms for inequalities that are valid for the 3-index assignment polytope. This idea could be fruitful in other problems as well. 
Of course, the improvement achieved for 3-index axial assignment problems arises from the fact that the corresponding formulation contains more variables than constraints. Therefore, analogous improvements could be plausible for other formulations which contain more variables than constraints. However, notice that for those problems where column generation is used to solve a linear programming formulation, this idea seems not applicable, since, in such a setting, variables are generated instead of violated inequalities. Thus, formulations with more variables than constraints that are not being solved by a column generation approach are susceptible to our idea.

\section{Acknowledgement}

We are thankful to Prof. Armin Fügenschuh for providing the routines to generate instances used in [18].

\section{References}

1. R. Alvarez-Valdes, F. Parreo and J.M. Tamarit, A branch-and-cut algorithm for the pallet loading problem, Computers and Operations Research, 32 (2005) 3007-3029.

2. G. Appa, D. Magos and I. Mourtos, On multi-index assignment polytopes, Linear Algebra Appl. 416 (2006) 224-241.

3. D. L. Applegate, R. E. Bixby, V. Chvátal and W. J. Cook, (2006), The Traveling Salesman Problem: A Computational Study, Princeton University Press, ISBN 978-0691-12993-8.

4. E. Balas and L. Qi, Linear time separation algorithms for the three index assignment polytope, Discrete Applied Mathematics 43 (1993) 1-12.

5. E. Balas and M. J. Saltzman, Facets of the three-index assignment polytope, Discrete Applied Mathematics 23 (1989) 201-229.

6. E. Balas and M. J. Saltzman, An algorithm for the three index assignment problem, Operations Research 39 (1991) 150-161.

7. E. Balas and M. Padberg, Set partitioning: a survey, SIAM Review 18(4) (1976) 710-760.

8. R Burkard and R Rudolf and G J Woeginger, Three-Dimensional Axial Assignment Problems with Decomposable Cost Coefficients, Discrete Applied Mathematics 65 (1996) 123-139.

9. A. Caprara and J.J. Salazar, Separating Lifted Odd-Hole Inequalities to Solve the Index Selection Problem, Discrete Applied Mathematics 92 (1999) 111-134.

10. E. Cheng and W.H.Cunningham, Wheel inequalities for stable set polytopes, Mathematical Programming 77 (1997) 389-421.

11. E. Cheng and W.H. Cunningham, Separation problems for the stable set polytope, pages 6579. The 4th Integer Programming and Combinatorial Optimization Conference Proceedings. 1995. E. Balas and J. Clausen (eds.).

12. Y. Crama and F.C.R. Spieksma, Approximation algorithms for three-dimensional assignment problems with triangle inequalities, European Journal of Operational Research, 60 (1992) 273-279.

13. L. Escudero, M. Landete and A. Marin, A branch-and-cut algorithm for the Winner Determination Problem, Decision Support Systems 46 (2009) 649-659.

14. L.K. Fleischer, A.N. Letchford and A. Lodi, Polynomial-time separation of a superclass of simple comb inequalities, Math. Oper. Res. 31 (2006) 696-713.

15. M. Grötschel and Y. Wakabayashi, A cutting plane algorithm for a clustering problem, Mathematical Programming 45 (1989) 59-96.

16. M. Grötschel and Y. Wakabayashi, Facets of the clique partitioning polytope, Mathematical Programming 47 (1990) 367-387. 


\begin{tabular}{|c|c|c|c|c|c|c|}
\hline Instance & $\mathrm{n}$ & iter & obj & no. of vio. ineq. & $\begin{array}{c}\text { Fast-Sep } \\
\text { time in sec }\end{array}$ & $\begin{array}{c}{[4]} \\
\text { time in sec }\end{array}$ \\
\hline $\begin{array}{c}\text { bs241 } \\
\text { Totaltime }\end{array}$ & 24 & $\begin{array}{l}0 \\
1 \\
2\end{array}$ & $\begin{array}{l}0.515936 \\
0.685279 \\
0.700093\end{array}$ & $\begin{array}{c}11 \text { (Type I) } \\
3 \text { (Type I) } \\
0\end{array}$ & $\begin{array}{l}<0.001 \\
<0.001 \\
<0.001 \\
<0.001\end{array}$ & $\begin{array}{l}0.016 \\
0.015 \\
0.015 \\
0.046\end{array}$ \\
\hline $\begin{array}{c}\text { bs242 } \\
\text { Totaltime }\end{array}$ & 24 & $\begin{array}{l}0 \\
1\end{array}$ & $\begin{array}{l}0 \\
0\end{array}$ & $\begin{array}{c}4 \text { (Type I) } \\
0\end{array}$ & $\begin{array}{l}<0.001 \\
<0.001 \\
<0.001\end{array}$ & $\begin{array}{c}<0.001 \\
0.015 \\
0.015\end{array}$ \\
\hline bs243 & 24 & $\begin{array}{l}0 \\
1 \\
2 \\
3\end{array}$ & $\begin{array}{l}0 \\
0 \\
0 \\
0\end{array}$ & $\begin{array}{c}2(\text { Type I) } \\
2 \text { (Type I) } \\
1 \text { (Type I) } \\
0\end{array}$ & $\begin{array}{l}<0.001 \\
<0.001 \\
<0.001 \\
<0.001 \\
<0.001\end{array}$ & $\begin{array}{c}0.016 \\
<0.001 \\
<0.001 \\
0.016 \\
0.032 \\
\end{array}$ \\
\hline $\begin{array}{c}\text { bs244 } \\
\text { Totaltime }\end{array}$ & 24 & $\begin{array}{l}0 \\
1 \\
2\end{array}$ & $\begin{array}{l}1 \\
1 \\
1\end{array}$ & $\begin{array}{c}4 \text { (Type I) } \\
1 \text { (Type I) } \\
0\end{array}$ & $\begin{array}{l}<0.001 \\
<0.001 \\
<0.001 \\
<0.001\end{array}$ & $\begin{array}{l}0.015 \\
0.016 \\
0.015 \\
0.046\end{array}$ \\
\hline $\begin{array}{c}\text { bs245 } \\
\text { Totaltime }\end{array}$ & 24 & $\begin{array}{l}0 \\
1 \\
2\end{array}$ & $\begin{array}{c}0.36903 \\
0.467105 \\
0.478788\end{array}$ & $\begin{array}{c}3 \text { (Type I) } \\
5 \text { (Type I) } \\
0\end{array}$ & $\begin{array}{l}<0.001 \\
<0.001 \\
<0.001 \\
<0.001\end{array}$ & $\begin{array}{c}<0.001 \\
0.016 \\
0.015 \\
0.031\end{array}$ \\
\hline $\begin{array}{c}\text { bs261 } \\
\text { Totaltime }\end{array}$ & 26 & $\begin{array}{l}0 \\
1 \\
2\end{array}$ & $\begin{array}{l}0 \\
0 \\
0\end{array}$ & $\begin{array}{c}4(\text { Type I) } \\
2(\text { Type I) } \\
0\end{array}$ & $\begin{array}{l}<0.001 \\
<0.001 \\
<0.001 \\
<0.001\end{array}$ & $\begin{array}{c}<0.001 \\
<0.001 \\
0.015 \\
0.015\end{array}$ \\
\hline Totaltime & 26 & $\begin{array}{l}0 \\
1 \\
2 \\
3\end{array}$ & $\begin{array}{l}0 \\
0 \\
0 \\
0\end{array}$ & $\begin{array}{l}6 \text { (Type I }) \\
2(\text { Type I) } \\
2(\text { Type I }) \\
0\end{array}$ & $\begin{array}{l}<0.001 \\
<0.001 \\
<0.001 \\
<0.001\end{array}$ & $\begin{array}{c}<0.001 \\
<0.001 \\
0.015 \\
0.031 \\
0.046\end{array}$ \\
\hline $\begin{array}{c}\text { bs263 } \\
\text { Totaltime }\end{array}$ & 26 & $\begin{array}{l}0 \\
1 \\
2\end{array}$ & $\begin{array}{l}1 \\
1 \\
1\end{array}$ & $\begin{array}{c}8(\text { Type I }) \\
1 \text { (Type I }) \\
0\end{array}$ & $\begin{array}{l}<0.001 \\
<0.001 \\
<0.001 \\
<0.001\end{array}$ & $\begin{array}{l}0.015 \\
0.015 \\
0.015 \\
0.045\end{array}$ \\
\hline $\begin{array}{c}\text { bs264 } \\
\text { Totaltime }\end{array}$ & 26 & $\begin{array}{l}0 \\
1\end{array}$ & $\begin{array}{l}0 \\
0\end{array}$ & $\begin{array}{c}3 \text { (Type I) } \\
0\end{array}$ & $\begin{array}{l}<0.001 \\
<0.001 \\
<0.001\end{array}$ & $\begin{array}{l}0.016 \\
0.031 \\
0.047\end{array}$ \\
\hline Totaltime & 26 & $\begin{array}{l}0 \\
1 \\
2 \\
3 \\
4\end{array}$ & $\begin{array}{c}0.304809 \\
0.368847 \\
0.378931 \\
0.38042 \\
0.380716\end{array}$ & $\begin{array}{c}7 \text { (Type I) } \\
3 \text { (Type I) } \\
1 \text { (Type I) } \\
1 \text { (Type I) } \\
0\end{array}$ & $\begin{array}{l}<0.001 \\
<0.001 \\
<0.001 \\
<0.001 \\
<0.001 \\
<0.001\end{array}$ & $\begin{array}{c}<0.001 \\
<0.001 \\
0.016 \\
<0.001 \\
0.031 \\
0.047\end{array}$ \\
\hline
\end{tabular}

Table 1 Computation times to find a violated clique inequality of type I and type II on instances from Balas and Saltzmann [6]

17. D.A.Grundel and P.M.Pardalos, Test Problem Generator for the Multidimensional Assignment Problem, Computational Optimization and Applications, 30(2) (2005) 133146.

18. B Höfler and A Fügenschuh, Parametrized GRASP Heuristics for Three-Index Assignment, EvoCOP, Lecture Notes in Computer science 3906 (2006) 61-72.

19. K. Kaparis and A.N. Letchford, Separation algorithms for 0-1 knapsack polytopes, Mathematical Programming 124 (2010) 69-91. 
20. J.D. Kececioglu, Hans-Peter Lenhof, K. Mehlhorn, P. Mutzel, K. Reinert, and M. Vingron, A polyhedral approach to sequence alignment problems. Discrete Applied Mathematics, 104(1-3) (2000) 143-186.

21. A.N. Letchford and M.M. Sørensen, Binary positive semidefinite matrices and associated integer polytopes. Mathematical Programming, 131(1)(2012) 253-271.

22. G.L. Nemhauser and L.A.Wolsey, Integer and Combinatorial Optimization, Wiley (1988).

23. M.Oosten, J.H.G.C. Rutten, and F.C.R. Spieksma, The clique partitioning problem: facets and patching facets, Networks 38, 209-226.

24. L. Qi and D. Sun, Polyhedral methods for solving three index assignment problems, in Nonlinear Assignment Problems: Algorithms and Applications, P.M. Pardalos and L. Pitsoulis, eds. (Kluwer Academic Publisher, Nowell, MA. USA, 2000) 91-107.

25. F. C. R. Spieksma, Multi-index assignment problems: complexity, approximation, applications, in Nonlinear Assignment Problems: Algorithms and Applications, P.M. Pardalos and L. Pitsoulis, eds. (Kluwer Academic Publisher, Nowell, MA. USA, 2000) pp. $1-12$.

26. J.M. Van den Akker, C.P.M. van Hoesel and M.W.P. Savelsbergh, A polyhedral approach to single machine scheduling problems, Mathematical Programming 85 (1999) 541-572.

27. S. Rebennack, M. Oswald, D. Oliver Theis, H. Seitz, G. Reinelt, and P.M. Pardalos, A Branch and Cut Solver for the Maximum Stable Set Problem, Journal of Combinatorial Optimization, 21(4) (2011) 434-457.

28. S. Ropke and J.F. Cordeau, Branch-and-Cut-and-Price for the Pickup and Delivery Problem with Time Windows, Transportation Science, 43 (2008) 267-286. 


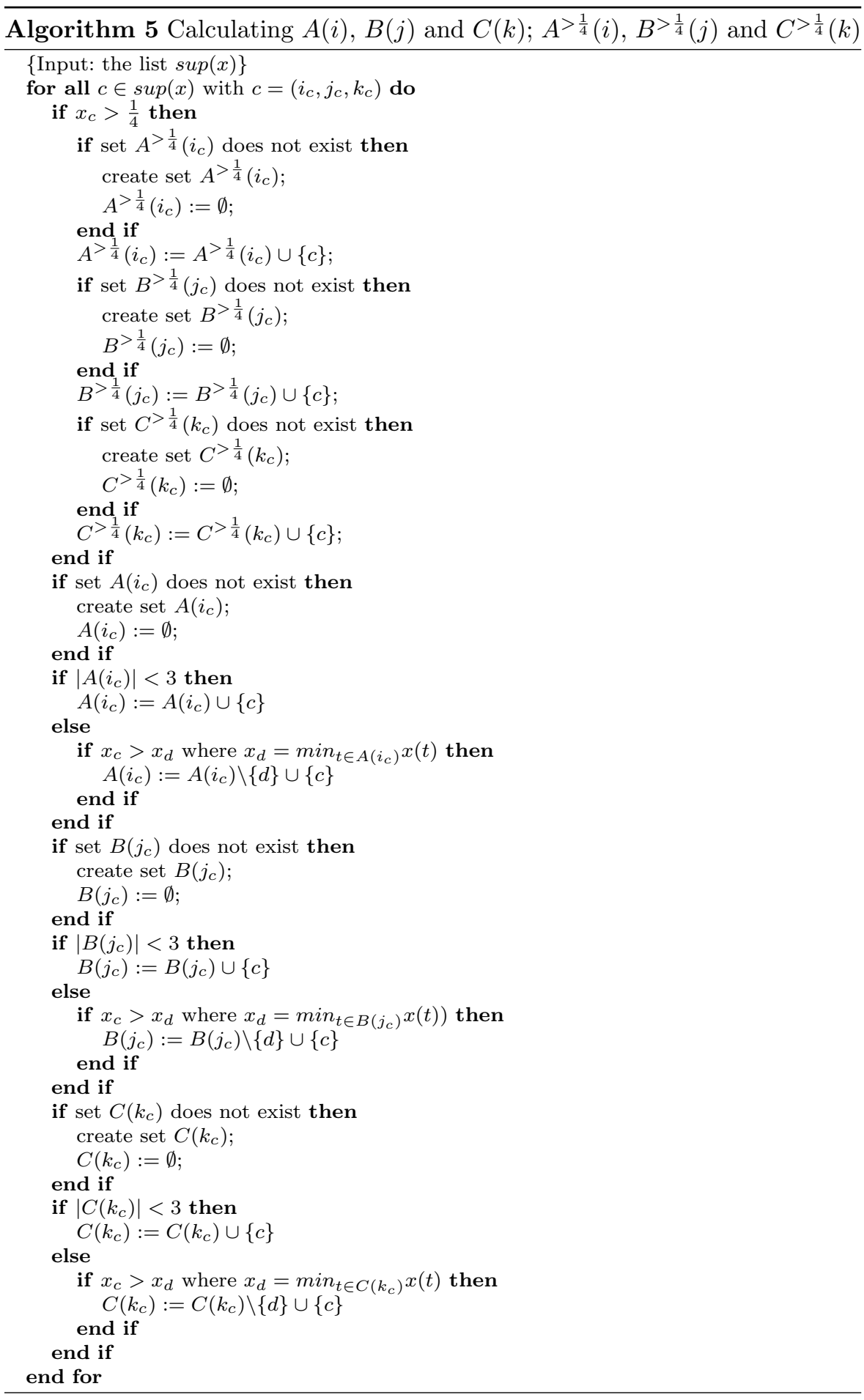




\begin{tabular}{|c|c|c|c|c|c|c|}
\hline Instance & $\mathrm{n}$ & iter & obj & no. of vio. ineq. & $\begin{array}{c}\text { Fast-Sep } \\
\text { time in sec }\end{array}$ & $\begin{array}{c}{[4]} \\
\text { time in sec }\end{array}$ \\
\hline Totaltime & 66 & $\begin{array}{l}0 \\
1 \\
2 \\
3 \\
4\end{array}$ & $\begin{array}{l}286 \\
286 \\
286 \\
286 \\
286\end{array}$ & $\begin{array}{l}1 \text { (Type I) } \\
1 \text { (Type I) } \\
4 \text { (Type I) } \\
2(\text { Type I) } \\
0\end{array}$ & $\begin{array}{l}0.015 \\
0.016 \\
0.015 \\
0.016 \\
0.031 \\
0.093\end{array}$ & $\begin{array}{c}0.297 \\
0.344 \\
0.36 \\
0.359 \\
1.157 \\
2.517\end{array}$ \\
\hline $\begin{array}{l}\text { 3D1299N1 } \\
\text { Totaltime }\end{array}$ & 33 & $\begin{array}{l}0 \\
1\end{array}$ & $\begin{array}{l}133 \\
133\end{array}$ & $\begin{array}{c}1 \text { (Type I) } \\
0\end{array}$ & $\begin{array}{l}<0.001 \\
<0.001 \\
<0.001\end{array}$ & $\begin{array}{l}0.015 \\
0.062 \\
0.077 \\
\end{array}$ \\
\hline $\begin{array}{l}\text { 3D1299N2 } \\
\text { Totaltime }\end{array}$ & 33 & $\begin{array}{l}0 \\
1 \\
2\end{array}$ & $\begin{array}{l}131 \\
131 \\
131\end{array}$ & $\begin{array}{c}2 \text { (Type I) } \\
1 \text { (Type I) } \\
0\end{array}$ & $\begin{array}{c}<0.001 \\
<0.001 \\
0.016 \\
0.016\end{array}$ & $\begin{array}{l}0.016 \\
0.016 \\
0.047 \\
0.079\end{array}$ \\
\hline $\begin{array}{l}\text { 3D1299N3 } \\
\text { Totaltime }\end{array}$ & 33 & $\begin{array}{l}0 \\
1\end{array}$ & $\begin{array}{l}131 \\
131\end{array}$ & $\begin{array}{c}1 \text { (Type I) } \\
0\end{array}$ & $\begin{array}{l}<0.001 \\
<0.001 \\
<0.001\end{array}$ & $\begin{array}{l}0.032 \\
0.047 \\
0.079\end{array}$ \\
\hline $\begin{array}{l}\text { 3DA99N2 } \\
\text { Totaltime }\end{array}$ & 33 & $\begin{array}{l}0 \\
1\end{array}$ & $\begin{array}{l}1400.5 \\
1400.5\end{array}$ & $\begin{array}{c}1 \text { (Type I) } \\
0\end{array}$ & $\begin{array}{l}<0.001 \\
<0.001 \\
<0.001\end{array}$ & $\begin{array}{c}<0.001 \\
0.047 \\
0.047\end{array}$ \\
\hline $\begin{array}{c}\text { 3DA198N3 } \\
\text { Totaltime }\end{array}$ & 66 & $\begin{array}{l}0 \\
1\end{array}$ & $\begin{array}{c}2758 \\
2758^{b}\end{array}$ & $\begin{array}{c}2 \text { (Type I) } \\
0\end{array}$ & $\begin{array}{c}0.016 \\
0 \\
0.016\end{array}$ & $\begin{array}{c}0.235 \\
0 \\
0.235\end{array}$ \\
\hline 3DI198N1 & 66 & $\begin{array}{l}0 \\
1 \\
2 \\
3 \\
4 \\
5\end{array}$ & $\begin{array}{l}9684 \\
9684 \\
9684 \\
9684 \\
9684 \\
9684\end{array}$ & $\begin{array}{l}8 \text { (Type I) } \\
6 \text { (Type I) } \\
1 \text { (Type I) } \\
3 \text { (Type I) } \\
1 \text { (Type I) } \\
0\end{array}$ & $\begin{array}{l}0.016 \\
0.016 \\
0.016 \\
0.015 \\
0.016 \\
0.015 \\
0.095\end{array}$ & $\begin{array}{l}0.235 \\
0.359 \\
0.359 \\
0.359 \\
0.281 \\
0.922 \\
2.515 \\
\end{array}$ \\
\hline $\begin{array}{l}\text { 3DI198N2 } \\
\text { Totaltime }\end{array}$ & 66 & $\begin{array}{l}0 \\
1 \\
2 \\
3\end{array}$ & $\begin{array}{l}8944 \\
8944 \\
8944 \\
8944\end{array}$ & $\begin{array}{c}1 \text { (Type I) } \\
1 \text { (Type I) } \\
2(\text { Type I) } \\
0\end{array}$ & $\begin{array}{c}0.016 \\
0 \\
0.016 \\
0.016 \\
0.048 \\
\end{array}$ & $\begin{array}{c}0.328 \\
0.328 \\
0.328 \\
1.156 \\
2.14 \\
\end{array}$ \\
\hline 3DIJ99N2 & 33 & $\begin{array}{l}0 \\
1\end{array}$ & $\begin{array}{l}5067 \\
5067\end{array}$ & $\begin{array}{l}5(\text { Type I }) \\
0(\text { Type I })\end{array}$ & $\begin{array}{l}<0.001 \\
<0.001 \\
<0.001\end{array}$ & $\begin{array}{l}0.016 \\
0.031 \\
0.047\end{array}$ \\
\hline 3DIJ99N3 & 33 & $\begin{array}{l}0 \\
1\end{array}$ & $\begin{array}{c}4286.5 \\
4287\end{array}$ & $\begin{array}{c}1 \text { (Type I) } \\
0\end{array}$ & $\begin{array}{c}0.015 \\
<0.001 \\
<0.001\end{array}$ & $\begin{array}{l}0.016 \\
0.047 \\
0.016\end{array}$ \\
\hline 3DI198N3 & 66 & $\begin{array}{l}0 \\
1 \\
2 \\
3\end{array}$ & $\begin{array}{l}9745 \\
9745 \\
9745 \\
9745\end{array}$ & $\begin{array}{c}11 \text { (Type I) } \\
3 \text { (Type I) } \\
3 \text { (Type I) } \\
0\end{array}$ & $\begin{array}{l}0.016 \\
0.020 \\
0.015 \\
0.016 \\
0.067\end{array}$ & $\begin{array}{l}0.281 \\
0.297 \\
0.344 \\
1.297 \\
2.219\end{array}$ \\
\hline
\end{tabular}

Table 2 Computation times to find a violated clique inequality of type I and type II for instances from Crama and Spieksma [12]; $b$ : integral solution 


\begin{tabular}{|c|c|c|c|c|c|c|}
\hline Instance & $\mathrm{n}$ & iter & obj & no. of vio. ineq. & $\begin{array}{c}\text { Fast-Sep } \\
\text { time in sec }\end{array}$ & $\begin{array}{c}{[4]} \\
\text { time in sec }\end{array}$ \\
\hline $\begin{array}{c}\text { quad66 } \\
\text { Totaltime }\end{array}$ & 66 & $\begin{array}{l}0 \\
1 \\
2 \\
3\end{array}$ & $\begin{array}{l}0 \\
0 \\
0 \\
0\end{array}$ & $\begin{array}{l}5(\text { Type I) } \\
3(\text { Type I) } \\
1(\text { Type I) } \\
0\end{array}$ & $\begin{array}{l}0.016 \\
0.015 \\
0.015 \\
0.031 \\
0.077\end{array}$ & $\begin{array}{l}0.297 \\
0.297 \\
0.281 \\
1.031 \\
1.906\end{array}$ \\
\hline $\begin{array}{c}\text { quad54 } \\
\text { Totaltime }\end{array}$ & 54 & $\begin{array}{l}0 \\
1 \\
2 \\
3 \\
4\end{array}$ & $\begin{array}{l}0 \\
0 \\
0 \\
0 \\
0\end{array}$ & $\begin{array}{l}6 \text { (Type I) } \\
2(\text { Type I) } \\
1 \text { (Type I) } \\
1 \text { (Type I }) \\
0\end{array}$ & $\begin{array}{l}0.015 \\
0.016 \\
0.016 \\
0.016 \\
0.016 \\
0.079 \\
\end{array}$ & $\begin{array}{c}0.125 \\
0.125 \\
0.109 \\
0.14 \\
0.593 \\
1.092\end{array}$ \\
\hline $\begin{array}{c}\text { quad25 } \\
\text { Totaltime }\end{array}$ & 25 & $\begin{array}{l}0 \\
1\end{array}$ & $\begin{array}{l}0 \\
0\end{array}$ & $\begin{array}{c}5 \text { (Type I) } \\
0\end{array}$ & $\begin{array}{l}<0.001 \\
<0.001 \\
<0.001\end{array}$ & $\begin{array}{c}<0.001 \\
0.031 \\
0.031\end{array}$ \\
\hline $\begin{array}{c}\text { quad } 80 \\
\text { Totaltime }\end{array}$ & 80 & $\begin{array}{l}0 \\
1 \\
2 \\
3\end{array}$ & $\begin{array}{l}0 \\
0 \\
0 \\
0\end{array}$ & $\begin{array}{c}6 \text { (Type I) } \\
3 \text { (Type I) } \\
1 \text { (Type I) } \\
0\end{array}$ & $\begin{array}{l}0.016 \\
0.016 \\
0.015 \\
0.031 \\
0.078 \\
\end{array}$ & $\begin{array}{l}0.625 \\
0.594 \\
0.579 \\
2.219 \\
4.017 \\
\end{array}$ \\
\hline $\begin{array}{c}\text { uniform10k25 } \\
\text { Totaltime }\end{array}$ & 25 & $\begin{array}{l}0 \\
1\end{array}$ & $\begin{array}{l}756.267296 \\
761.836735\end{array}$ & $\begin{array}{c}5 \text { (Type I) } \\
0\end{array}$ & $\begin{array}{l}<0.001 \\
<0.001 \\
<0.001\end{array}$ & $\begin{array}{c}<0.001 \\
0.016 \\
0.016\end{array}$ \\
\hline $\begin{array}{c}\text { uniform10k40 } \\
\text { Totaltime }\end{array}$ & 40 & $\begin{array}{l}0 \\
1 \\
2 \\
3\end{array}$ & $\begin{array}{l}564.645488 \\
569.201393 \\
569.405369 \\
569.477446\end{array}$ & $\begin{array}{c}4 \text { (Type I) } \\
2 \text { (Type I) } \\
2(\text { Type II }) \\
0\end{array}$ & $\begin{array}{c}<0.001 \\
0.015 \\
<0.001 \\
0.016 \\
0.031\end{array}$ & $\begin{array}{l}0.016 \\
0.015 \\
0.125 \\
0.125 \\
0.281\end{array}$ \\
\hline $\begin{array}{l}\text { uniform10k54 } \\
\text { Totaltime }\end{array}$ & 54 & $\begin{array}{l}0 \\
1 \\
2\end{array}$ & $\begin{array}{l}399.862191 \\
400.863177 \\
400.982559\end{array}$ & $\begin{array}{c}5 \text { (Type I) } \\
1 \text { (Type I) } \\
0\end{array}$ & $\begin{array}{l}<0.001 \\
0.015 \\
0.015 \\
0.030 \\
\end{array}$ & $\begin{array}{c}0.11 \\
0.11 \\
0.5 \\
0.72 \\
\end{array}$ \\
\hline $\begin{array}{c}\text { uniform10k66 } \\
\text { Totaltime }\end{array}$ & 66 & $\begin{array}{l}0 \\
1 \\
2\end{array}$ & $\begin{array}{l}339.595966 \\
340.944483 \\
341.010642\end{array}$ & $\begin{array}{c}7 \text { (Type I) } \\
1 \text { (Type I) } \\
0\end{array}$ & $\begin{array}{l}0.016 \\
0.016 \\
0.016 \\
0.048\end{array}$ & $\begin{array}{l}0.297 \\
0.281 \\
1.078 \\
1.656\end{array}$ \\
\hline $\begin{array}{l}\text { uniform10k80 } \\
\text { Totaltime }\end{array}$ & 80 & $\begin{array}{l}0 \\
1 \\
2\end{array}$ & $\begin{array}{l}228.865278 \\
229.326601 \\
229.355625\end{array}$ & $\begin{array}{c}5 \text { (Type I) } \\
1 \text { (Type I) } \\
0\end{array}$ & $\begin{array}{l}0.016 \\
0.016 \\
0.047 \\
0.079\end{array}$ & $\begin{array}{l}0.609 \\
0.563 \\
2.313 \\
3.485\end{array}$ \\
\hline
\end{tabular}

Table 3 Computation times to find a violated clique inequality of type I and type II for instances from Höfler and Fügenschuh [18] 


\begin{tabular}{|c|c|c|c|c|c|c|}
\hline Instance & $\mathrm{n}$ & iter & obj & no. of vio. ineq. & $\begin{array}{c}\text { Fast-Sep } \\
\text { time in sec }\end{array}$ & $\begin{array}{c}{[4]} \\
\text { time in sec }\end{array}$ \\
\hline $\begin{array}{c}\text { brw66 } \\
\text { Totaltime }\end{array}$ & 66 & $\begin{array}{l}0 \\
1 \\
2 \\
3\end{array}$ & $\begin{array}{l}5759 \\
5759 \\
5759 \\
5759\end{array}$ & $\begin{array}{c}8 \text { (Type I) } \\
8 \text { (Type I) } \\
2(\text { Type I) } \\
0\end{array}$ & $\begin{array}{l}0.031 \\
0.016 \\
0.015 \\
0.025 \\
0.087\end{array}$ & $\begin{array}{l}0.297 \\
0.297 \\
0.297 \\
1.156 \\
2.047\end{array}$ \\
\hline $\begin{array}{c}\text { brw80 } \\
\text { Totaltime }\end{array}$ & 80 & $\begin{array}{l}0 \\
1 \\
2 \\
3 \\
4\end{array}$ & $\begin{array}{l}6911 \\
6911 \\
6911 \\
6911 \\
6911\end{array}$ & $\begin{array}{l}9 \text { (Type I) } \\
9 \text { (Type I) } \\
4(\text { Type I) } \\
2(\text { Type I) } \\
0(\text { Type I) }\end{array}$ & $\begin{array}{l}0.016 \\
0.031 \\
0.031 \\
0.032 \\
0.047 \\
0.157\end{array}$ & $\begin{array}{l}0.563 \\
0.547 \\
0.547 \\
0.516 \\
2.421 \\
4.617 \\
\end{array}$ \\
\hline $\begin{array}{c}\text { brw54 } \\
\text { Totaltime }\end{array}$ & 54 & $\begin{array}{l}0 \\
1 \\
2 \\
3 \\
4\end{array}$ & $\begin{array}{l}5004 \\
5004 \\
5004 \\
5004 \\
5004\end{array}$ & $\begin{array}{l}5 \text { (Type I }) \\
7 \text { (Type I }) \\
2(\text { Type I }) \\
2(\text { Type I }) \\
0\end{array}$ & $\begin{array}{c}0.016 \\
<0.001 \\
<0.001 \\
0.015 \\
0.016 \\
0.047\end{array}$ & $\begin{array}{c}0.11 \\
0.125 \\
0.109 \\
0.109 \\
0.485 \\
0.938\end{array}$ \\
\hline $\begin{array}{c}\text { brw25 } \\
\text { Totaltime }\end{array}$ & 25 & $\begin{array}{l}0 \\
1 \\
2\end{array}$ & $\begin{array}{l}2211 \\
2211 \\
2211\end{array}$ & $\begin{array}{c}4 \text { (Type I }) \\
2(\text { Type I }) \\
0\end{array}$ & $\begin{array}{l}<0.001 \\
<0.001 \\
<0.001 \\
<0.001\end{array}$ & $\begin{array}{c}0.015 \\
<0.001 \\
0.016 \\
0.031\end{array}$ \\
\hline $\begin{array}{l}\text { cluster66 } \\
\text { Totaltime }\end{array}$ & 66 & $\begin{array}{l}0 \\
1 \\
2\end{array}$ & $\begin{array}{l}0 \\
0 \\
0\end{array}$ & $\begin{array}{c}6(\text { Type I) } \\
6 \text { (Type I) } \\
0\end{array}$ & $\begin{array}{l}0.016 \\
0.018 \\
0.015 \\
0.048\end{array}$ & $\begin{array}{l}0.297 \\
0.312 \\
1.094 \\
1.703\end{array}$ \\
\hline $\begin{array}{l}\text { cluster54 } \\
\text { Totaltime }\end{array}$ & 54 & $\begin{array}{l}0 \\
1 \\
2 \\
3\end{array}$ & $\begin{array}{l}0 \\
0 \\
0 \\
0\end{array}$ & $\begin{array}{c}3 \text { (Type I) } \\
2 \text { (Type I) } \\
1 \text { (Type I) } \\
0\end{array}$ & $\begin{array}{c}0.016 \\
0.015 \\
<0.001 \\
0.016 \\
0.047\end{array}$ & $\begin{array}{l}0.109 \\
0.125 \\
0.125 \\
0.485 \\
0.844\end{array}$ \\
\hline $\begin{array}{l}\text { cluster25 } \\
\text { Totaltime }\end{array}$ & 25 & $\begin{array}{l}0 \\
1 \\
2\end{array}$ & $\begin{array}{c}1.635417 \\
1.744681 \\
1.75\end{array}$ & $\begin{array}{c}2 \text { (Type I) } \\
3 \text { (Type I) } \\
0\end{array}$ & $\begin{array}{c}0.016 \\
<0.001 \\
<0.001 \\
0.016\end{array}$ & $\begin{array}{c}<0.001 \\
0.015 \\
0.188 \\
0.203\end{array}$ \\
\hline cluster80 & 80 & $\begin{array}{l}0 \\
1 \\
2 \\
3\end{array}$ & $\begin{array}{l}0 \\
0 \\
0 \\
0\end{array}$ & $\begin{array}{c}8 \text { (Type I) } \\
7 \text { (Type I) } \\
2(\text { Type I }) \\
0\end{array}$ & $\begin{array}{l}0.015 \\
0.015 \\
0.031 \\
0.031 \\
0.092\end{array}$ & $\begin{array}{l}0.594 \\
0.594 \\
0.594 \\
2.344 \\
4.126\end{array}$ \\
\hline
\end{tabular}

Table 4 Computation times to find a violated clique inequality of type I and type II for instances from Höfler and Fügenschuh [18] 


\begin{tabular}{|c|c|c|c|c|c|c|}
\hline \hline Instance & $\mathrm{n}$ & iter & obj & $\begin{array}{c}\text { Fast-Sep } \\
\text { no. of vio. ineq. }\end{array}$ & $\begin{array}{c}{[4]} \\
\text { time in sec }\end{array}$ & time in sec \\
\hline n100d3prob1 & 100 & 0 & 473.000001 & 44 (Type I) & 0.028 & 1.219 \\
& & 1 & 474 & 29 (Type I) & 0.016 & 1.297 \\
& & 2 & $474^{b}$ & 0 & 0 & 0 \\
& & & 0 & 0.044 & 2.516 \\
\hline n100d3prob2 & 100 & 0 & 472.000001 & 38 (Type I) & 0.046 & 1.391 \\
& & 1 & 472.000001 & 25 (Type I) & 0.047 & 1.422 \\
& & 2 & 472.007813 & 11 (Type I) & 0.047 & 1.281 \\
& & 4 & 473 & 4 (Type I) & 0.047 & 1.391 \\
& & 4 & 473 & 1 (Type I) & 0.046 & 1.484 \\
& & & 473 & 0 & 0.062 & 1.406 \\
& & & 0 & 0.295 & 8.375 \\
\hline
\end{tabular}

Table 5 Computation times to find a violated clique inequality of type I and type II for instances from [17]; $b$ : integral solution

\begin{tabular}{|c|c|c|c|c|}
\hline Instance & & $\begin{array}{c}\text { Fast-Sep } \\
\text { time in sec }\end{array}$ & $\begin{array}{c}{[4]} \\
\text { time in sec }\end{array}$ & $\begin{array}{c}\text { Lp } \\
\text { time in sec }\end{array}$ \\
\hline Cluster54 & 54 & 0.047 & 0.844 & 0.079 \\
Cluster66 & 66 & 0.048 & 1.703 & 0.641 \\
Cluster80 & 80 & 0.092 & 4.126 & 0.843 \\
quad54 & 54 & 0.079 & 1.092 & 0.297 \\
quad66 & 66 & 0.077 & 1.906 & 0.344 \\
quad80 & 80 & 0.078 & 1.000 & 4.017 \\
brw54 & 54 & 0.047 & 0.138 & 0.490 \\
brw66 & 66 & 0.057 & 2.047 & 0.876 \\
brw80 & 80 & 0.157 & 4.617 & 1.834 \\
uniform10k54 & 54 & 0.030 & 0.720 & 0.203 \\
uniform10k66 & 66 & 0.048 & 1.656 & 0.562 \\
uniform10k80 & 80 & 0.079 & 3.485 & 1.235 \\
n100d3prob1 & 100 & 0.044 & 2.516 & 186.197 \\
n100d3prob2 & 100 & 0.295 & 8.375 & $>250$ \\
\hline
\end{tabular}

Table 6 Comparison of total separation times with LP solve times after iteration 1 